\title{
REZENSIONEN
}

\section{Parlamentarische Kontrolle der Außenpolitik}

Pilz, Volker: Der Auswärtige Ausschuss des Deutschen Bundestages und die Mitwirkung des Parlaments an der auswärtigen und internationalen Politik, Duncker \& Humblot (Beiträge zum Parlamentsrecht, Band 65), Berlin 2007, 201 Seiten, € 68,-.

Nachdem sich Volker Pilz gemeinsam mit Ekkehard Münzing in einer ersten Studie für diese Zeitschrift mit dem Auswärtigen Ausschuss beschäftigt hat ${ }^{1}$, ist nun seine hier anzuzeigende rechtswissenschaftliche Dissertation über den Auswärtigen Ausschuss erschienen, die bei Wolfgang Zeh an der Deutschen Hochschule für Verwaltungswissenschaften in Speyer entstand. Die Studie ist interdisziplinär angelegt: Historische, juristische und politikwissenschaftliche Inhalte werden zu einem lesenswerten Gesamtbild zusammengefügt. Erfreulicherweise beschränkt sich Pilz nicht auf den Auswärtigen Ausschuss des Bundestages, sondern geht bei der Behandlung auswärtiger Angelegenheiten durch das Parlament bis zu den Reichstagen in der Kaiserzeit und der Weimarer Zeit zurück. So gelingt es ihm, die Entstehung einiger parlamentarischer Usancen aufzuzeigen (zum Beispiel „geschlossener Ausschuss" S. 50; Selbstbefassungsrecht S. 89) oder aber auch erfolgreicher Legendenbildung entgegenzuwirken. Pilz weist beispielsweise nach, dass der spätere Bundespräsident Theodor Heuss schon in einem Beitrag von 1933 Friedrich Naumann zu unrecht das Verdienst zuschreibt, die Einrichtung des Ausschusses für Auswärtige Angelegenheiten angeregt zu haben. Naumann kann keinesfalls als dessen „spiritus rector“ bezeichnet werden, sondern ist allenfalls als „Antragsteller“ anzusehen (S. 32 ff., S. 35).

Pilz zeichnet detailliert die Entwicklung parlamentarischer Befassung mit der Außenpolitik im Auswärtigen Ausschuss nach, der im Unterschied zu den meisten anderen Ausschüssen kaum an der Gesetzgebung des Bundestages beteiligt ist und allenfalls völkerrechtliche Verträge behandelt. Ausgewertet wurden neben der einschlägigen Fachliteratur insbesondere die Reichstagsprotokolle sowie die Sitzungsprotokolle des Auswärtigen Ausschusses, die seit August 1919 maschinenschriftlich vorliegen. Bedauerlich ist, dass aus der 10. bis 12. Wahlperiode des Bundestages zahlreiche Ausschussprotokolle fehlen (S. 24) und gar seit Herbst 1999 nur Beschlussprotokolle verfasst werden. Pilz vermutet, dass seinerzeit „offensichtlich mehr Wert auf die Außendarstellung des Ausschusses gelegt [wurde] als auf das zeitaufwendige Anfertigen von Sitzungsprotokollen“ (S. 86, Fn. 92). Hinter dieser Bemerkung verbirgt sich Kritik in mehrerlei Hinsicht: Geltungsbedürfnisse der Mitglieder des Ausschusses werden einer ordentlichen Protokollführung entgegengesetzt, und für zukünftige Historiker werden vergleichbare Studien für die letzten Jahre der „Bonner Republik“ und die Phase der Wiedervereinigung nicht möglich sein, weil die Entstehung eines Mei-

1 Volker Pilz / Ekkehard Münzing, Der Auswärtige Ausschuß des Deutschen Bundestages: Aufgaben, Organisation und Arbeitsweise, in: ZParl, 29. Jg. (1998), H. 4, S. 575 ff. 
nungsbildes unter den Abgeordneten im Ausschuss nicht mehr zuverlässig rekonstruiert werden kann. Es ist vorstellbar, dass das Fehlen von Protokollen sich schon dann bemerkbar machen wird, wenn aus tagespolitischen Gründen - wie es ja oft passiert - die Hintergründe und Grundlagen für eine Entscheidung des Bundestages zuverlässig verifiziert werden müssen.

Pilz' Buch ist kein Ersatz für eine Geschichte des Auswärtigen Ausschusses (so Pilz selbst, S. 25), sondern hat die heutige Rolle des Bundestages und seines Auswärtigen Ausschusses in der auswärtigen und internationalen Politik im Blick (S. 26). Mit großem Ertrag fließen in die Arbeit Ergebnisse aus Gesprächen mit Abgeordneten, Mitarbeitern der Fraktionen, der Bundestagsverwaltung (hier dem Ausschusssekretariat) und des Auswärtigen Amts ein. Zur Charakterisierung des Einflusses der Parlamentarier in der auswärtigen Politik streut Pilz gelegentlich Anekdoten ein, wie über Carlo Schmids Trinkfestigkeit bei dem Besuch Konrad Adenauers 1955 in Moskau oder den Bericht über Hans Sterckens Aufenthalt im Sudan 1989, als dieser in einer Direktübertragung im sudanesischen Fernsehen dem durch Putsch ins Amt gelangten Militärdiktator zurief: „Mr. President [...] don't kill your people.“ (S. 127 f.) Mit gleichem Kurzweil liest sich von gelegentlich geäußerten despektierlichen Vorurteilen, der Auswärtige Ausschuss sei aufgrund seines hohen Anteils an verdienten Altparlamentariern und ehemaligen Ministern ein „Altersruhesitz“ oder „Elefantenfriedhof". Pilz widerlegt auch die Behauptung von Marieluise Beck, der Auswärtige Ausschuss sei ein Ausschuss mit „viel Renommee und wenig Arbeit“ (S. 19), und bewertet seine Bedeutung im Vergleich mit dem Haushaltsausschuss oder dem Ausschuss für Wirtschaft und Arbeit, der immerhin ein zentrales gesellschaftspolitisches Feld zu bearbeiten hat. Er gesteht zu, dass der Haushaltsausschuss das „Königsrecht“ des Parlaments wahrnimmt und mit Geld Politik gemacht wird (S. 176), doch betont er, dass der Auswärtige Ausschuss die Entscheidungen des Bundestages über Krieg und Frieden vorbereitet. Erfreulich ist auch die handbuchartige Darstellung der Mitwirkung des Bundestages in interparlamentarischen Gremien wie in der IPU, der Parlamentarischen Versammlung des Europarates, der WEU, NATO und OSZE.

Pilz kann für sich mit dieser Arbeit in Anspruch nehmen, einen originären Beitrag zu einem bedeutenden Thema der Demokratie geleistet zu haben: der parlamentarischen Kontrolle der Außenpolitik.

Michael F. Feldkamp

\section{Rituale in Parlamenten}

Crewe, Emma und Marion G. Müller (Hrsg.): Rituals in Parliaments. Political, Anthropological and Historical Perspectives on Europe and the United States, Peter Lang Verlag, Frankfurt am Main u.a. 2006, 209 Seiten, € 39,-.

Der Frage, ob parlamentarische Rituale von Bedeutung sind, widmet sich der vorliegende Band, der auf englischsprachigen Beiträgen einer Konferenz im Warburg-Haus Hamburg basiert. Es ist ein explorativ angelegtes Buch, das nicht beabsichtigt, eine neue einheitliche Theorie oder einen eigenständigen Theoriebeitrag zu leisten, sondern das Potential für in- 